EGU2020-4858

https://doi.org/10.5194/egusphere-egu2020-4858

EGU General Assembly 2020

(c) Author(s) 2020. This work is distributed under

the Creative Commons Attribution 4.0 License.

\title{
Understanding the 2017 warm event in North Tropical Atlantic
}

\author{
Ana Trindade ${ }^{1,2}$, Marta Matín-Rey ${ }^{2}$, Marcos Portabella ${ }^{2}$, Eleftheria Exarchou ${ }^{3}$, Pablo Ortega ${ }^{3}$, and \\ Iñigo Gómara ${ }^{4}$ \\ ${ }^{1}$ Universitat Politecnica de Catalunya (UPC), Barcelona, Spain (atrindade@icm.csic.es) \\ ${ }^{2}$ Institut de Ciències del Mar (ICM), CSIC, Barcelona, Spain \\ ${ }^{3}$ Barcelona Supercomputing Center (BSC), Barcelona, Spain \\ ${ }^{4}$ Universidad Complutense de Madrid (UCM), Madrid, Spain
}

The Atlantic Ocean has suffered tremendous warming during recent decades as a consequence of anthropogenic forcing, modulated by the natural low frequency variability. Special attention should be paid to the high temporal frequency of warm interannual events in the North Tropical Atlantic (NTA) since the early 2000s, resulting in the most intense hurricane seasons on record (Hallam et al., 2017; Lim et al., 2018; Murakami et al., 2018; Klotzbach et al., 2018; Camp et al., 2018). Moreover, NTA sea surface temperature anomalies during boreal spring have been suggested as a potential precursor to the Equatorial Mode (Foltz and McPhaden, 2010ab; Burmeister et al., 2016; Martín-Rey and Lazar, 2019; Martín-Rey et al., 2019).

This study aims to investigate the development of the 2017 NTA spring-summer warming event, which was the strongest of the last decade, as well as the importance of an accurate ocean forcingin the simulation of this event. For such purpose, a set of four simulations using distinct ocean wind forcing products, namely from the EC-Earth model, ERA-Interim (ERAi) reanalysis and a new ERAi-corrected ocean wind product (ERAstar), have been performed and analysed.The latter consists of average geolocated scatterometer-based corrections applied to ERAi output (Trindade et al., 2019).In this sense, ERAstar includes some of the physical processes missing or misrepresented by ERA-i, and corrects for large-scale NWP parameterization and dynamical errors.

The air-sea processes underlying the onset and development of the warm 2017 NTA event and the wave activity present in the equatorial Atlantic will be explored to determine their possible connection with the equatorial sea surface temperature variability. Furthermore, the comparison between the different experiments allows us to validate the new surface wind dataset and evaluate the importance of accurate, high-resolution ocean forcing in the representation of tropical Atlantic variability. 